\title{
Affective personality differences in neural processing efficiency confirmed using fMRI
}

\author{
JEREMY R. GRAY \\ Yale University, New Haven, Connecticut \\ GREGORY C. BURGESS \\ Washington University, St. Louis, Missouri \\ ALEXANDRE SCHAEFER \\ Yale University, New Haven, Connecticut \\ and \\ TAL YARKONI, RANDY J. LARSEN, and TODD S. BRAVER \\ Washington University, St. Louis, Missouri
}

\begin{abstract}
To test for a relation between individual differences in personality and neural-processing efficiency, we used functional magnetic resonance imaging (fMRI) to assess brain activity within regions associated with cognitive control during a demanding working memory task. Fifty-three participants completed both the self-report behavioral inhibition sensitivity (BIS) and behavioral approach sensitivity (BAS) personality scales and a standard measure of fluid intelligence (Raven's Advanced Progressive Matrices). They were then scanned as they performed a three-back working memory task. A mixed blocked/ event-related fMRI design enabled us to identify both sustained and transient neural activity. Higher BAS was negatively related to event-related activity in the dorsal anterior cingulate, the lateral prefrontal cortex, and parietal areas in regions of interest identified in previous work. These relationships were not explained by differences in either behavioral performance or fluid intelligence, consistent with greater neural efficiency. The results reveal the high specificity of the relationships among personality, cognition, and brain activity. The data confirm that affective dimensions of personality are independent of intelligence, yet also suggest that they might be interrelated in subtle ways, because they modulate activity in overlapping brain regions that appear to be critical for task performance.
\end{abstract}

Some important aspects of the human psyche are consistently revealed in brain function, but not in overt behavior (Wilkinson \& Halligan, 2004). The central focus of this article is one such endophenotype-namely, neural-processing efficiency, or the relative ease with which someone performs a given information-processing task. Individuals who need to exert greater mental effort to perform a task or compensate for stressful circumstances (Hockey, 1997) can be said to have lower efficiency. The key individual differences may not be revealed in overt behavior (performance), despite real and important differences in the facility with which that performance level is achieved (i.e., efficiency). Relatedly, one function of the prefrontal cortex may be to exert compensatory control over behavior when additional de-

The research was supported by grants from the National Science Foundation and the National Institute of Mental Health. Some of these data have been published in abstract form (J. R. Gray \& G. C. Burgess, Journal of Research in Personality, 2004, 38, 35-36) and in a full report (J. R. Gray, C. F. Chabris, \& T. S. Braver, 2003, Nature Neuroscience, 6, 316-322). Correspondence concerning this article should be addressed to J. R. Gray, Psychology Department, Yale University, Box 208205, New Haven, CT 06520 (e-mail: jeremy.gray@yale.edu). mands are imposed (Braver et al., 1997; Bunge, Klingberg, Jacobsen, \& Gabrieli, 2000). In this research, we used functional magnetic resonance imaging (fMRI) to study individual differences in personality, compensatory control, and neural efficiency during a cognitive load.

M. W. Eysenck and Calvo (1992) elaborated the idea of cognitive-processing efficiency, doing so to account for exceptions in the literature on the effects of trait anxiety on performance. Anxious individuals often have lower performance (especially on difficult tasks), but not always. Why should there be exceptions? M. W. Eysenck and Calvo drew a key distinction between performance effectiveness and processing efficiency. Effectiveness refers to an objectively measurable level of performance, such as the percentage of questions answered correctly on a test. Efficiency refers to the ratio between effectiveness and the amount of effort needed to attain the criterion level of performance. According to M. W. Eysenck and Calvo, anxiety impairs processing efficiency more than it impairs performance effectiveness, thereby leaving open whether performance is actually impaired or not on a given occasion. High-anxious individuals are hypothesized to be less efficient but can compensate by expending additional effort on the task. This hypothesis 
holds considerable explanatory potential. A practical difficulty with testing the cognitive-processing efficiency model, however, is that one of its central constructs, mental effort, cannot be measured directly in behavioral performance. To overcome the difficulty, one possibility is to use physiological indicators of effort (M. W. Eysenck \& Calvo, 1992; Fairclough \& Houston, 2004; J. R. Gray \& Braver, 2002).

A potentially useful physiological index of mental effort is task-related activity within working memory networks, including the dorsal anterior cingulate cortex (ACC) and the lateral prefrontal cortex (PFC; J. R. Gray $\&$ Braver, 2002). The dorsal ACC is part of the network of brain areas that support cognitive control (e.g., Bush, Luu, \& Posner, 2000; Devinsky, Morrell, \& Vogt, 1995; MacDonald, Cohen, Stenger, \& Carter, 2000; Paus, 2001; Paus, Koski, Caramanos, \& Westbury, 1998). Activation in the dorsal ACC has been reported for complex or difficult cognitive tasks (Bush et al., 2000; Devinsky et al., 1995; Paus, 2001; Paus et al., 1998), and for this reason, appears to be related to mental effort. Interestingly, activity in the ACC has been linked to peripheral psychophysiological responses (Critchley, Mathias, \& Dolan, 2001), and such peripheral measures are also used to index mental effort (e.g., Fairclough \& Houston, 2004). The lateral PFC is also part of a wider network subserving cognitive control. The magnitude of dorsolateral PFC activity varies parametrically with working memory load at a group level (Braver et al., 1997), and activity in the ventrolateral and dorsolateral PFC increases with performance differences, as tested at an individual level (Rypma \& D'Esposito, 1999). Finally, individual differences in fluid intelligence predict brain activity in the lateral PFC during a working memory task (J. R. Gray, Chabris, \& Braver, 2003).

Although recent investigations of personality have often focused on individual differences in affective processes (Canli, Sivers, Whitfield, Gotlib, \& Gabrieli, 2002; Costa \& McCrae, 1980; Larsen \& Ketelaar, 1991), there is also theoretical and empirical work focusing on differences in information processing, including working memory (Humphreys \& Revelle, 1984). Several recent studies illustrate this emphasis. For example, extraversion correlated positively with performance on a verbal $n$-back task (Lieberman \& Rosenthal, 2001), which is known to recruit the PFC and the central executive component of working memory (Braver et al., 1997). Of note, extraverts performed better only under conditions that imposed a high cognitive load (e.g., multitasking, but not single tasks), consistent with a processing efficiency explanation. Under conditions of low cognitive load, introverts may be able to compensate for lower processing efficiency by increased exertion of mental effort, but as task difficulty increases, such compensation reaches its limits. Extraverts also had better performance in a Sternberg task (Lieberman, 2000) and a prospective memory task (Heffernan \& Ling, 2001), both of which recruit the PFC.
Our focus is on two personality traits that we refer to as behavioral approach sensitivity (BAS) and behavioral inhibition sensitivity (BIS; Carver, Sutton, \& Scheier, 2000; Carver \& White, 1994; J. A. Gray, 1994; Sutton \& Davidson, 1997). In theory, BIS represents individual differences in sensitivity to cues of threat (threats induce behavioral inhibition and withdrawal), and BAS represents individual differences in sensitivity to cues of reward (rewards induce behavioral approach). Personality theorists are essentially unanimous that two major personality dimensions are associated with differences in emotional processing (Costa \& McCrae, 1980; J. A. Gray, 1994; Larsen \& Ketelaar, 1991; Zelenski \& Larsen, 1999). These are typically called extraversion and neuroticism, although some theorists call them $B A S$ and $B I S$, or positive affect and negative affect. Certainly, BAS and BIS are related to extraversion and neuroticism, although the exact relation is debated. Although it is perhaps not the dominant view, Carver et al. (2000) have argued that BIS and BAS may represent what is most fundamental about neuroticism and extraversion. We are interested in possible relations between individual differences in sensitivity to cues of reward (BAS) and cognitive control processes, with the guiding intuition that individual differences in goal-directed (reward-seeking) processes might relate to differences in the cognitive and neural mechanisms that support goal-directed behavior (i.e., cognitive control, including working memory).

In several theories of extraversion, the concept of arousal is used to explain the personality-cognition relationships. This account is based on two assumptions. H. J. Eysenck (1967) suggested that extraverts and introverts were mainly differentiated by their basal arousal level, extraverts having a lower arousal level than introverts do. And it is posited that the effects of arousal on cognitive performance conform to an inverted-U function (Yerkes \& Dodson, 1908), in which moderate levels of arousal have a beneficial effect on performance, whereas low or high levels have a detrimental effect, especially in complex cognitive tasks (Humphreys \& Revelle, 1984). The consequence is that introverts are expected to perform better than extraverts in low-arousal tasks but to be outperformed by extraverts in high-arousal tasks. A more contemporary version of the arousal account has been provided by Lieberman and Rosenthal (2001), who proposed linking the concept of arousal with catecholamine activity - more particularly, to dopamine and norepinephrine. Norepinephrine is more related to wakefulness and anxiety, and dopamine to positive affect and novelty detection. Intriguingly, dopamine is released during demanding tasks (Aalto, Bruck, Laine, Nagren, \& Rinne, 2005).

There are few neuroimaging studies of personality and processing efficiency, although several imaging studies have been performed to examine personality and activity during cognitive tasks (e.g., Ebmeier et al., 1994; Kumari, ffytche, Williams, \& Gray, 2004). We recently investigated the effects of personality on working-memory- 
related activity, using a block design fMRI study (J. R. Gray \& Braver, 2002). In 14 participants, we found a negative correlation between activity in the dorsal ACC (also called the caudal ACC) and BAS. In addition, we found marginal relations between ACC activity and BIS. Behaviorally, in 129 participants, we found a weak, positive relationship between BAS and $n$-back performance $(r=.18$, $p<.05$ ), with a larger effect size in the 14 participants who were scanned ( $r=.27)$. Despite significant results, a limitation of the fMRI study was the small sample size.

Individual differences in neural-processing efficiency have also been related to cognitive variation and, in particular, have been hypothesized to help explain the relation between intelligence and brain activity (Haier, Siegel, Tang, Abel, \& Buchsbaum, 1992). On the basis of a negative correlation between psychometric intelligence and cerebral glucose metabolic rate, Haier and colleagues suggested that greater neural efficiency should help individuals achieve higher scores on intelligence tests. Other work has suggested that whether high-IQ individuals show a pattern of greater neural efficiency depends on participant sex and task content (Neubauer, Fink, \& Schrausser, 2002), and positive correlations have also been found (J. R. Gray et al., 2003; Haier, White, \& Alkire, 2003). Negative relations could reflect behavioral differences (working longer on a task should lead to greater activity) and so, by themselves, do not unambiguously imply differences in neural efficiency specifically. Importantly, psychometric intelligence has been consistently related to the speed of information processing - for example, as assessed by inspection time (for reviews, see Deary, 2000; J. R. Gray \& Thompson, 2004). Moreover, inspection time is supported by brain activity in two networks (Deary et al., 2004), one of which is apparently related to cognitive control (based on localization to regions associated with cognitive control). Thus, prior work suggests that, at a minimum, it may be important to assess and control for psychometric intelligence when investigating personality differences in neural-processing efficiency. A third dimension of personality (as assessed using the NEO-PI), openness to experience, has been consistently related to intelligence and cognitive function, so much so that it is increasingly referred to as openness/ intellect (DeYoung, Peterson, \& Higgins, in press).

The aim of this article is to extend empirical work on personality, mental effort, and processing efficiency at a neural level. To do so, we will follow up and extend a conceptually similar investigation in our previous study (J. R. Gray \& Braver, 2002). The present report replicates this study closely but goes beyond it by (1) considering affective and cognitive variables simultaneously, controlling for individual differences in fluid intelligence and behavioral performance when assessing personality effects, (2) using a mixed state-item design, to assess both transient and sustained neural activity, (3) examining regions outside of the ACC that also support cognitive control and working memory, and (4) doing so in a large fMRI sample $(n=53)$. The two key empirical questions we will address are the following. Does the negative correlation between BAS and workingmemory-related activity hold in areas known to be critical for working memory and cognitive control? And is this relationship not explained by differences in behavioral performance or fluid intelligence? We sought and found clear evidence for both questions, strongly suggesting that higher BAS is related to less mental effort and greater neural-processing efficiency. Psychometrically, our overall approach is very strong: an initial exploratory investigation in a first data set (J. R. Gray \& Braver, 2002), followed by a confirmatory investigation in an independent data set (the present study).

\section{METHOD}

\section{Participants}

The participants were healthy, right-handed, native English speakers ( $n=60,29$ male; ages, 18-37 years) from Washington University and the surrounding community, screened to exclude a history of neurological disorder, current psychoactive medication, or factors contraindicating fMRI. All the participants gave written informed consent. None had participated in our previous study (J. R. Gray \& Braver, 2002); that is, the samples were completely independent. The experiment was approved by the Washington University Medical Center Human Subjects Committee. Of the 60 participants who completed the protocol, some had fMRI data sets that were compromised by excessive head movement, technical problems, unacceptably low behavioral performance, or too few correct trials for estimating event-related responses. This left $n=53$ for one set of analyses (sustained state-related activity and transient item-related activity) and $n=48$ for a second set of analyses (in which item-related activity was further classified into trial types with varying degrees of difficulty).

\section{Procedure and Design}

To assess variation in personality, we used the 20 -item self-report BIS-BAS scales (Carver \& White, 1994) and EPQ-R (S. B. G. Eysenck, H. J. Eysenck, \& Barrett, 1985), administered prior to the fMRI session.

To assess variation in cognitive ability, we used the Raven's Advanced Progressive Matrices (APM; Raven, Raven, \& Court, 1998), a standard test of fluid intelligence, also administered prior to the fMRI session. Fluid intelligence was taken to be the number of correct responses on APM Set II (36 questions) made in 40 min, after practice on three items from APM Set I.

To assess variation in neural activity, we used fMRI to assess activity as the participants performed a three-back working memory task that was administered using PsyScope (Cohen, MacWhinney, Flatt, \& Provost, 1993) on a Macintosh G3. The three-back task consisted of viewing a series of stimuli. The instructions were to indicate, for each stimulus, whether it matched or did not match the stimulus seen previously exactly three stimuli back (three back). Responses were made using fiber-optic response keys. To respond with high accuracy, the participants had to keep track of several stimuli at once, every few seconds updating which stimuli were relevant. Most of the participants found this challenging, but not overly so. For a given scanning run, the stimuli were either all words (concrete English nouns) or all faces (unfamiliar, male and female intermixed; J. R. Gray, Braver, \& Raichle, 2002). The stimuli were shown one per trial for $2.0 \mathrm{sec}$, with a fixation point (crosshair) shown between stimuli (for $0.36 \mathrm{sec}$ ). Each scanning run had 4 unanalyzed fixation trials, followed by four blocks of 21 task trials (16 task stimuli with 5 crosshair fixation trials, $2.36 \mathrm{sec}$ each, ran- 
domly interspersed to introduce temporal jitter) and by $23.6 \mathrm{sec}(10$ trials) of resting fixation (a dash), for a total of 128 trials per scanning run (2.36 sec per trial).

\section{MR Image Acquisition and Analysis}

Localizer images, a high-resolution structural image (MPRAGE), and a series of functional images were collected on a Siemens 1.5T Vision system. Functional (echo planar) images consisted of 16 axial slices, each $8 \mathrm{~mm}$ thick with a $3.75 \times 3.75 \mathrm{~mm}$ in-plane resolution.

Data from two scanning runs per participant are reported here (one run with words, one run with faces, order counterbalanced), although additional runs were also obtained. Just prior to each scanning run, the participants watched one of six 7-min videos, two of which were emotionally neutral and four of which were emotionally evocative (order counterbalanced). The videos were included as part of another investigation focusing on the emotional conditions. All the imaging data reported here are from the two neutral conditions.

On the basis of our prior work, we had specific functional-anatomic predictions, including both the direction of correlations and which brain regions should show them. We therefore opted to conduct region-of-interest (ROI) analyses exclusively, using ROIs from published work. This strategy allows a direct and conservative replication, while also remaining sensitive to potentially weak effects (controlling for false positive results by having specific predictions, rather than by correcting for multiple comparisons across the whole brain). Because we were also interested in the potential relation with intelligence, we examined activity within four ROIs that had been defined previously within the present data set $(n=48)$ on the basis of their relation to fluid intelligence (J. R. Gray et al., 2003): in the bilateral lateral PFC, Brodmann Areas 44/45/46, left 21 voxels at -461824 and right 65 voxels at 44921 , and in the bilateral parietal cortex, BA 40, left 8 voxels at $-56-4233$ and right 12 voxels at $46-3345$ (see Figure 1A). These were the only regions that plausibly mediated the relation between fluid intelligence and working memory performance and, thus, were especially interesting to examine in regard to mental effort, processing efficiency, and compensatory control. We also examined four subregions in the ACC, using the same regions as those defined previously in a fully independent sample $(n=14 ; \mathrm{J}$. R. Gray \& Braver, 2002). The four ACC regions (Figure 1B) were defined on the basis of comparing three-back task activity against a resting fixation, as showing either predicted activation (caudal/dorsal ACC, 110 voxels centered on Talairach coordinate 22945 ) or predicted deactivation (posteriorrostral ACC, 47 voxels at 2224 12; rostral ACC, 83 voxels at 242 9; subgenual ACC, 26 voxels at 23626 ).

Specifically, we predicted that we would replicate our previously observed negative correlation between BAS and working-memoryrelated activity in the dorsal ACC and the four ROIs related to fluid intelligence, but not in the other three ACC subregions. We were agnostic as to whether the relation should hold in item-related activity, state-related activity, or both.

The data were preprocessed and analyzed in several stages prior to the extraction of activation values from the ROIs. After movement and artifact correction, functional images were normalized within each scanning run and temporally aligned within each brain volume. Functional images were resampled into $3-\mathrm{mm}$ isotropic voxels, transformed into atlas space (Talairach \& Tournoux, 1988), and smoothed with a Gaussian filter (9-mm FWHM). We then constructed two general linear models (GLMs) for each participant, each estimating the activity at each voxel. The first GLM was created to include state and item regressors for face and word conditions separately, allowing us to statistically separate neural signals that were sustained across the task block (state) from those that were transient (item; Braver, Reynolds, \& Donaldson, 2003; Donaldson, Petersen, Ollinger, \& Buckner, 2001).
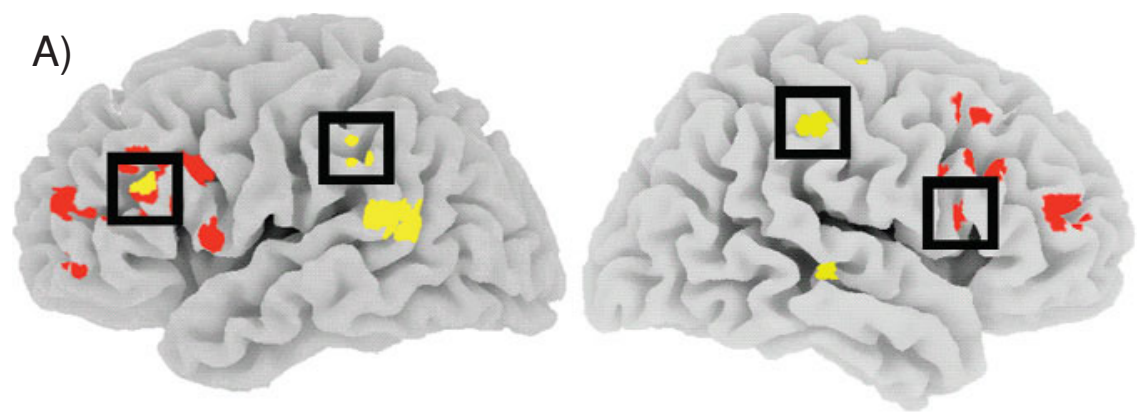

B)



Figure 1. Regions of interest in (A) the lateral prefrontal and parietal cortex, shown in lateral views of the left and right hemispheres (left on the left); and (B) the anterior cingulate cortex shown in a sagittal view, anterior to the left; here, caudal is synonymous with dorsal. Panel $A$ is adapted from J. R. Gray, Chabris, and Braver, copyright 2003 by the Nature Publishing Group. Panel B is adapted from J. R. Gray and Braver (2002). 
Table 1

Personality Predicts Working-Memory-Related Activity

\begin{tabular}{|c|c|c|c|c|c|c|c|c|c|}
\hline \multirow{2}{*}{$\begin{array}{l}\text { Region of } \\
\text { Interest }\end{array}$} & \multirow[b]{2}{*}{ Center } & \multicolumn{2}{|c|}{ BAS } & \multicolumn{2}{|c|}{ BIS } & \multicolumn{2}{|c|}{ E } & \multicolumn{2}{|c|}{$\mathrm{N}$} \\
\hline & & State & Item & State & Item & State & Item & State & Item \\
\hline \multicolumn{10}{|l|}{ Lateral prefrontal } \\
\hline & $-46,18,24$ & -.01 & $\begin{array}{l}-.42^{*} \\
\left(-.41^{*}\right)\end{array}$ & .19 & .02 & -.12 & $-.38 *$ & .02 & -.09 \\
\hline R BA 44/45 & $44,9,21$ & .09 & $\begin{array}{l}-.27^{*} \\
\left(-.26^{*}\right)\end{array}$ & .11 & -.21 & -.10 & -.18 & .10 & -.26 \\
\hline \multicolumn{10}{|l|}{ Parietal } \\
\hline L BA 40 & $-56,-42,33$ & .07 & $\begin{array}{l}-.42^{*} \\
\left(-.42^{*}\right)\end{array}$ & .11 & .14 & -.04 & $-.39^{*}$ & .27 & -.11 \\
\hline R BA 40 & $46,-33,45$ & $\begin{array}{l}.38^{*} \\
\left(.38^{*}\right)\end{array}$ & $\begin{array}{l}-.31^{*} \\
\left(-.31^{*}\right)\end{array}$ & .09 & -.05 & .14 & $-.35^{*}$ & .26 & -.03 \\
\hline \multicolumn{10}{|l|}{ Anterior cingulate } \\
\hline Dorsal BA 24 & $-2,9,45$ & .06 & $\begin{array}{l}-.28^{*} \\
\left(-.27^{*}\right)\end{array}$ & .20 & .07 & .01 & $-.30^{*}$ & .22 & .02 \\
\hline Posterior rostral & $-2,24,12$ & .08 & .04 & $.30^{+}$ & -.08 & -.09 & .01 & $.32^{*}$ & -.05 \\
\hline Rostral & $2,42,9$ & .08 & .07 & $\begin{array}{l}.41^{*} \\
\left(.41^{*}\right)\end{array}$ & -.16 & -.20 & .04 & $.35^{*}$ & -.10 \\
\hline Subgenual & $2,36,-6$ & .10 & $\begin{array}{c}.28^{+} \\
\left(.28^{+}\right)\end{array}$ & .16 & -.14 & .04 & .14 & $.28^{+}$ & -.19 \\
\hline
\end{tabular}

Note $-N=53$. Prefrontal and parietal regions were defined on the basis on fluid intelligence (J. R. Gray, Chabris, \& Braver, 2003); anterior cingulate regions were defined on the basis of working-memory-related activity in an independent data set (J. R. Gray \& Braver, 2002). Values are zero-order correlations $(d f=51)$ between individual difference variables (BAS, BIS, $\mathrm{E}, \mathrm{N}$ ) and brain activity (state, item). The values in parentheses are the weakest of three partial correlations $(d f=50)$ after controlling for fluid intelligence (APM), accuracy on the threeback task $\left(d^{\prime}\right)$, and average response time for correct trials on the three-back task. Center, center of region-of-interest mass in Talairach coordinates (Talairach \& Tournoux, 1988; see Figure 1); BA, Brodmann area; BAS, behavioral approach sensitivity; BIS, behavioral inhibition sensitivity; E, extraversion; N, neuroticism; L, left; R, right. ${ }^{*} p<.05$, corrected for multiple comparisons. ${ }^{+} p<.05$, not corrected for multiple comparisons.

To permit a more detailed analysis in terms of trial types that varied in the demand for cognitive control, the second GLM was similar but had three types of items, rather than one generic item type. In a previous study involving the $n$-back task and RAPM scores (J. R. Gray et al., 2003), strong differences in behavioral performance demonstrated that different $n$-back trials vary considerably in difficulty. This effect suggested a differential relation to the demand for cognitive control: higher demand on lure trials (nontarget trials with a two- four- or five-back match, in which a strong spurious sense of familiarity led to high interference), low demand on nonlure trials (low-interference nontarget trials with no previous match or with a match that was not temporally proximal to the three-back item), and intermediate demand on target trials (three-back match).

Both GLMs gave estimates of the magnitudes of the state and item activity, indexed in terms of the percentage of change in the MR signal. The magnitude estimates were then averaged over all voxels within a given ROI and then over stimulus type. The resulting average values were then correlated with individual difference variables.

\section{RESULTS}

\section{Behavioral Data}

The distributions of BIS and BAS scores, which were consistent with those from other samples (Carver \& White, 1994; J. R. Gray \& Braver, 2002), showed good variability: BIS scores, $M=20.4, S D=3.99$, range of 10-27; BAS scores, $M=40.7, S D=5.8$, range of 26-52. APM scores were above the general population average $(M=26.0, S D=4.27$, range of 11-34), also with a good range. As was expected, the participants'
BIS, BAS, and APM scores were uncorrelated $(r=-.06$ for APM with BAS, $r=-.04$ for APM with BIS).

There was no correlation between BAS and mean accuracy in the working memory task (assessed in terms of the signal detection measure, $d^{\prime} ; r=-.03$ ) or between BAS and mean response time (RT; $r=-.19, p \mathrm{~s}>.10)$.

The intercorrelations among personality variables were as expected, with positive relations between BAS and $\mathrm{E}(r=.56, p<.001)$, and BIS and $\mathrm{N}(r=.59, p<$ $.001)$ and a marginal correlation between BIS and $\mathrm{E}(r=$ $-.24, p=.087$; all other $p s>.15)$.

\section{Neuroimaging Data}

State- and item-related activity. As was predicted, BAS was negatively correlated with item-related activity in the five a priori ROIs related to cognitive control (the dorsal ACC, the bilateral PFC, and bilateral BA 40; see Table 1). The negative correlation indicates lower itemrelated activation for participants with higher BAS scores. There were negative correlations between BAS and activity in the left lateral PFC, the left parietal lobe, the right parietal lobe, and the right lateral PFC. Confirming our previous findings, BAS correlated negatively with activity in the dorsal ACC. When formally testing for differences in the strength of correlations (adjusting for the fact that these correlation coefficients are not statistically independent; see Meng, Rosenthal, \& Rubin, 1992), the BAS-activity relation was more 
strongly negative in the dorsal ACC than in the other three ACC subregions (all $p \mathrm{~s}<.05)$. Although BAS correlated positively with item-related activity in the subgenual ACC (representing less item-related deactivation for higher BAS), this correlation was not predicted a priori and did not survive a Bonferroni correction for the six comparisons ( 3 ACC subregions $\times 2$ activity types). On the other hand, some correlations that were not predicted a priori nonetheless survived correction for multiple comparisons. Specifically, BIS correlated positively with state-related activity in the rostral ACC (less sustained deactivation for higher BIS), and BAS was positively correlated with state activity in right BA 40 .

Critically, the above relationships between BAS and item activity (in the dorsal ACC, the bilateral PFC, and the bilateral parietal lobe and between BIS and state activity (in the rostral ACC) were not explained by behavioral performance or fluid intelligence. Specifically, the partial correlations between personality (BAS, BIS) and task-related activity, when controlling for APM scores (fluid intelligence), accuracy, or RT, were either the same or larger than the zero-order correlations (see Table 1).

Activity on specific trial types. As was shown previously, behavioral performance on the $n$-back task demonstrated that some of the trials were more difficult than other trials, with lure trials imposing a markedly higher demand on cognitive control than target or nonlure trials did. Therefore, we investigated whether the relationship between BAS and transient (item) activity would also be specific to one trial type or whether it would hold generally across all trial types.

The correlation with BAS did not hold selectively to the lure trials, the condition with the highest demand for cognitive control (see Table 2). BAS was related to lure trial activity but was also related to activity on other trial types. BAS predicted transient neural activity (at significant or marginally significant levels) in 8 of 12 tests (4 regions $\times 3$ activity type combinations). These relationships persisted, even after controlling for APM scores,

Table 2

Personality and Specific Trial Types

\begin{tabular}{|c|c|c|c|c|c|c|}
\hline \multirow{2}{*}{$\begin{array}{l}\text { Region of } \\
\text { Interest }\end{array}$} & \multicolumn{3}{|c|}{ BAS } & \multicolumn{3}{|c|}{ BIS } \\
\hline & Lure & Target & Non & ure & Target & Nonlur \\
\hline \multicolumn{7}{|c|}{ Lateral prefrontal } \\
\hline & $-.29^{+}$ & -.2 & -.3 & -.09 & -.06 & .02 \\
\hline R BA & $-.37^{*}$ & - . & & & .01 & .18 \\
\hline \multicolumn{7}{|l|}{ Parietal } \\
\hline L B & $-.29^{+}$ & .02 & -.1 & -.10 & -.20 & -.14 \\
\hline R BA 40 & -.16 & $-.28^{+}$ & $-.29^{*}$ & -.03 & -.02 & .07 \\
\hline
\end{tabular}

Notes-Regions are the same as those in Table 1. In all regions, APM correlated positively with lure trial activity and weakly, if at all, with nonlure activity (see J. R. Gray, Chabris, \& Braver, 2003, Table 1). Values are zeroorder correlations $(n=48$ [see the text], $d f=46)$. Regions and abbreviations are the same as those in Table 1. Lure trials, high demand for cognitive control; target, intermediate demand; nonlure, lower demand (see text). ${ }^{*} p<.05$, corrected for multiple comparisons. ${ }^{+} p<.05$, not corrected for multiple comparisons. for accuracy, or for RT. BIS had no relation to activity on specific trial types.

Activity estimates from a block design analysis. To facilitate comparison with our prior report (J. R. Gray \& Braver, 2002), we also analyzed the present data in a block design, rather than state-item, analysis. The dorsal ACC-BAS correlation held slightly more strongly $(r=$ $-.37, p=.006)$, whereas none of the other ACC regions correlated significantly with BAS (all $r \mathrm{~s}<.16, p \mathrm{~s}>$ .25).

Correlations with extraversion and neuroticism. Extraversion was related to brain activity in qualitatively the same way as BAS (see Table 1). Moreover, neuroticism was related to activity in much the same way as BIS (Table 1).

\section{DISCUSSION}

The results provide clear answers to the two empirical questions we started with. First, BAS was indeed associated with greater task-related activity in all five theoretically relevant brain regions - namely, the bilateral lateral PFC, the bilateral parietal cortex, and the dorsal ACC. Moreover, these results also held when individual differences in behavioral performance and fluid intelligence were controlled for statistically. The specificity of these effects directly implies that the results are not simply an artifact of high-BAS individuals being less willing to work at the task (and so engaged these neural networks less strongly, which would also be revealed in behavioral differences); it also implies that it is not the case that high-BAS individuals were simply more intelligent and so did not need to recruit these networks as strongly to achieve the same level of behavioral performance. Thus, the overall pattern of results strongly indicates BAS-related individual differences in neural processing efficiency and argue against a differential recruitment of mechanisms that are otherwise equivalently efficient (e.g., due to motivation, intelligence, and other nonspecific factors).

The data also bear on the relationship between two major dimensions of human individual differences: affective aspects of personality and intelligence (cf. Bates \& Rock, 2004; Fink, Schrausser, \& Neubauer, 2004). On the one hand, they confirm that personality and intelligence are independent, because they had no correlation and made independent contributions to lateral PFC and parietal activity. Not only was BAS independent of $\mathrm{gF}$, but also the correlations in the PFC and the parietal cortex were in opposite directions: High $\mathrm{gF}$ was related to stronger event-related activity, whereas high BAS was related to weaker activity. Yet by the same token, the two constructs might be interrelated (cf. Fink et al., 2004), because they modulate activity in overlapping brain regions that appear critical for task performance.

The observed correlation with BAS in the dorsal ACC $(r=-.28$, item-related activity) was in the same direction as, albeit weaker than, the correlation we observed previ- 
ously ( -.63 in the neutral conditions, block design). Although the previous $95 \%$ confidence interval $(r=-.87$ to -.18 ) includes the present value, the weaker effect here could also reflect increased susceptibility to noise in the state-item design. The dorsal ACC-BAS correlation was indeed stronger when a block design analysis was conducted $(r=-.37)$, instead of the state-item analysis, yet it was still weaker than that in our previous report. This underscores the value of conducting confirmatory (replication) studies, and of using large samples.

The BAS effect did not hold exclusively on trials imposing the strongest demand on cognitive controlnamely, the lure trials. Instead, the neural-processing efficiency associated with high-BAS individuals effectively held across all trial types - not uniformly, but clearly lacking the strong specificity to the lure trials that we observed for fluid intelligence in these same participants (J. R. Gray et al., 2003). One possibility is that even the nonlure and target trials impose some demand on cognitive control, but just less than the lure trials. Indeed, behaviorally, accuracy on target and nonlure trials was also significantly correlated with fluid intelligence $(r \mathrm{~s}=$ .29-.36), whereas brain activity on those trials was not.

To speculate, a possible mechanistic explanation of the BAS-activity relationship derives from a recent theoretical account of extraversion, based on the neurotransmitter dopamine. Depue and Collins (1999) have argued that individual differences in extraversion are due to variation in a network of dopaminergic neurons involving the ventral tegmental area, the nucleus accumbens, and projections to the cortex. Dopamine function may modulate executive control by protecting or updating representations of goal states that lead to reward (Ashby, Isen, \& Turken, 1999; Braver \& Cohen, 2000). Therefore, higher extraversion may relate to more efficient executive control abilities, resulting from their common relationship with midbrain DA function. Brain areas belonging to the dopaminergic circuit are more activated in extraverts than in introverts during positive emotions (Canli et al., 2002). In addition, reward significantly increased working-memory-related activation in the dorsal lateral prefrontal cortex during an $n$-back task (Pochon et al., 2002) and was related to dorsal ACC activity during a decision-making task (Bush et al., 2002). Thus dopaminergic systems are a more specific candidate for further investigation of personality-neuralprocessing efficiency relations.

Our results may also have implications for H. J. Eysenck's (1967) theory of extraversion. Although BAS and extraverion are different constructs in theory, in practice, their measures are highly redundant (Rusting \& Larsen, 1999; Zelenski \& Larsen, 1999). Contrary to Eysenck's original hypothesis that extraverts have lower resting arousal levels, EEG studies suggest that extraverts do not show a reduced level of cortical arousal at rest (Gale, 1986). However, in studies in which nervous system responsiveness to moderate levels of stim- ulation has been examined, extraverts have consistently shown smaller or slower responses than introverts have (Bullock \& Gilliland, 1993; Gale, 1983). Consequently, extraversion appears to be related primarily to differences in nervous system reactivity, rather than to a tonic level of arousal (Green, 1984; Stelmack, 1990). Our results are consistent with this hypothesis, because highBAS individuals showed a smaller magnitude of hemodynamic response than low-BAS individuals did to the 3-back working memory task. Although it remains an open question to what extent this relationship between cerebral blood flow and extraversion holds true for other cortical regions and other forms of stimulation, that we observed this relation in our paradigm is consistent with the proposal that extraversion effects are strongest during goal-directed behavior.

Our results are opposite in direction from those reported by Kumari et al. (2004), in which higher extraversion scores (as assessed by a slightly different measure than the one we used; H. J. Eysenck \& S. B. G. Eysenck, 1991) predicted greater activity during an $n$-back task in 11 participants. Kumari and colleagues interpreted their result in terms of Eysenck's theory, as representing a lower baseline level in extraverts, from which they can become more strongly activated (i.e., greater differences between baseline and task). Although this interpretation is consistent with their results, it is not consistent with the present interpretation of Eysenck's theory, that higher extraversion is best described as being associated with lower arousability (smaller movements from baseline), rather than with lower arousal levels (lower baselines; e.g., Green, 1984; Stelmack, 1990). We cannot be entirely sure of the reason for the discrepancy between the results, although the differences between personality scales are not likely responsible. The present study has several interpretive strengths, being a replication with a large sample in a priori ROIs, while controlling for behavioral variables (including task performance and intelligence), and state-item design.

We also found evidence that a second dimension of personality, BIS, is related to sustained activity within the rostral (and perhaps the posterior-rostral) ACC. Although not predicted a priori, this result survived correction for multiple comparisons and accords well with previous work, including PET studies of resting brain activity and individual differences in trait negative affect (Zald, Mattson, \& Pardo, 2002) and explicit manipulations of anxiety (Simpson, Drevets, Snyder, Gusnard, \& Raichle, 2001). The same result held for neuroticism.

In sum, for BAS (and extraversion), the data provide strong support for the hypothesis of personality differences in neural-processing efficiency. The data confirm and extend our previous findings (J. R. Gray \& Braver, 2002) by having a large sample and more nuanced measures and by including other relevant brain regions. More broadly, the data have implications for biological theories of personality and suggest that understanding the 
function of the prefrontal, anterior cingulate, and parietal cortex will require understanding individual differences in both affective and cognitive variables.

\section{REFERENCES}

Aalto, S., Bruck, A., Laine, M., Nagren, K., \& Rinne, J. O. (2005). Frontal and temporal dopamine release during working memory and attention tasks in healthy humans: A positron emission tomography study using the high-affinity dopamine $\mathrm{d} 2$ receptor ligand [11c]flb 457. Journal of Neuroscience, 25, 2471-2477.

Ashby, F. G., Isen, A. M., \& Turken, A. U. (1999). A neuropsychological theory of positive affect and its influence on cognition. $P s y-$ chological Review, 106, 529-550.

BAtes, T. C., \& Rock, A. (2004). Personality and information processing speed: Independent influences on intelligent performance. Intelligence, 32, 33-46.

Braver, T. S., \& Cohen, J. D. (2000). On the control of control: The role of dopamine in regulating prefrontal function and working memory. In S. Monsell \& J. Driver (Eds.), Attention and performance XVIII: Control of cognitive processes (pp. 713-738). Cambridge, MA: MIT Press.

Braver, T. S., Cohen, J. D., Nystrom, L. E., Jonides, J., Smith, E. E., \& NolL, D. C. (1997). A parametric study of prefrontal cortex involvement in human working memory. NeuroImage, 5, 49-62.

Braver, T. S., Reynolds, J. R., \& Donaldson, D. I. (2003). Neural mechanisms of transient and sustained cognitive control during task switching. Neuron, 39, 713-726.

BulLoCK, W. A., \& GilLILAND, K. (1993). Eysenck's arousal theory of introversion-extraversion: A converging measures investigation. Journal of Personality \& Social Psychology, 64, 113-123.

Bunge, S. A., Klingberg, T., Jacobsen, R. B., \& Gabrieli, J. D. E. (2000). A resource model of the neural basis of executive working memory. Proceedings of the National Academy of Sciences, 97, 3573-3578.

Bush, G., LuU, P., \& Posner, M. I. (2000). Cognitive and emotional influences in anterior cingulate cortex. Trends in Cognitive Sciences, $\mathbf{4}$, 215-222.

Bush, G., Vogt, B. A., Holmes, J., Dale, A. M., Greve, D., \& Jenike, M. A. (2002). Dorsal anterior cingulate cortex: A role in rewardbased decision making. Proceedings of the National Academy of Sciences, 99, 507-512.

Canli, T., Sivers, H., Whitfield, S. L., Gotlib, I. H., \& Gabrieli, J. D. E. (2002). Amygdala response to happy faces as a function of extraversion. Science, 296, 2191

Carver, C. S., Sutton, S. K., \& Scheier, M. F. (2000). Action, emotion, and personality: Emerging conceptual integration. Personality \& Social Psychology Bulletin, 26, 741-751.

CARver, C. S., \& White, T. (1994). Behavioral inhibition, behavioral activation, and affective responses to impending reward and punishment: The bis/bas scales. Journal of Personality \& Social Psychologv, 67, 319-333.

Cohen, J., MacWhinney, B., Flatt, M., \& Provost, J. (1993). PsyScope: An interactive graphic system for designing and controlling experiments in the psychology laboratory using Macintosh computers. Behavior Research Methods, Instruments, \& Computers, 25 257-271.

Costa, P. T., \& McCrae, R. R. (1980). Influence of extraversion and neuroticism on subjective well-being: Happy and unhappy people. Journal of Personality \& Social Psychology, 38, 668-678.

Critchley, H. D., Mathias, C. J., \& Dolan, R. J. (2001). Neural activity in the human brain relating to uncertainty and arousal during anticipation. Neuron, 29, 537-545.

DeARY, I. J. (2000). Looking down on human intelligence. New York: Oxford University Press.

Deary, I. J., Simonotto, E., Meyer, M., Marshall, A., Marshall, I., GodDARD, N., \& WARDLAW, J. M. (2004). The functional anatomy of inspection time: An event-related fMRI study. NeuroImage, 22, 1466-1479.
Depue, R. A., \& Collins, P. F. (1999). Neurobiology of the structure of personality: Dopamine, facilitation of incentive motivation, and extraversion. Behavioral \& Brain Sciences, 22, 491-569.

Devinsky, O., Morrell, M. J., \& Vogt, B. (1995). Contributions of anterior cingulate cortex to behavior. Brain, 118, 279-306.

DeYoung, C. G., Peterson, J. B., \& Higgins, D. M. (in press). Sources of openness/intellect: Cognitive and neuropsychological correlates of the fifth factor of personality. Journal of Personality.

Donaldson, D. I., Petersen, S. E., Ollinger, J. M., \& Buckner, R. L. (2001). Dissociating item and state components of recognition memory using fMRI. NeuroImage, 13, 129-142.

Ebmeier, K. P., Deary, I. J., O'Carroll, R. E., Prentice, N., MofFOOT, A. P. R., \& Goodwin, G. M. (1994). Personality associations with the uptake of the cerebral blood flow marker 99mtc-exametazime estimated with single photon emission tomography. Personality \& Individual Differences, 17, 587-595.

EYSENCK, H. J. (1967). The biological basis of personality. Springfield, IL: Thomas.

Eysenck, H. J., \& Eysenck, S. B. G. (1991). Manual of the Eysenck personality scales. London: Hodder \& Stoughton.

EysencK, M. W., \& Calvo, M. G. (1992). Anxiety and performance: The processing efficiency theory. Cognition \& Emotion, 6, 409-434

Eysenck, S. B. G., Eysenck, H. J., \& Barrett, P. (1985). A revised version of the psychoticism scale. Personality \& Individual Differences, $\mathbf{6}, 21-29$

FaIRCLOUgh, S. H., \& Houston, K. (2004). A metabolic measure of mental effort. Biological Psychology, 66, 177-190.

Fink, A., Schrausser, D. G., \& Neubauer, A. C. (2004). The moderating influence of extraversion on the relationship between IQ and cortical activity. Personality \& Individual Differences, 33, 311-326.

GaLE, A. (1983). Electroencephalographic studies of extraversionintroversion: A case study in the psychophysiology of individual differences. Personality \& Individual Differences, 4, 371-380.

GaLE, A. (1986). Extraversion-introversion and spontaneous rhythms of the brain: Retrospect and prospect. In J. Strelau, F. Farley, \& A. Gale (Eds.), The biological basis of personality and behavior: Psychophysiology, performance, and applications (Vol. 2, pp. 25-42). Washington, DC: Hemisphere.

Gray, J. A. (1994). Personality dimensions and emotion systems. In P. Ekman \& R. J. Davidson (Eds.), The nature of emotion (pp. 329331). New York: Oxford University Press.

Gray, J. R., \& Braver, T. S. (2002). Personality predicts workingmemory-related activation in the caudal anterior cingulate cortex Cognitive, Affective, \& Behavioral Neuroscience, 2, 64-75.

Gray, J. R., Braver, T. S., \& Raichle, M. E. (2002). Integration of emotion and cognition in the lateral prefrontal cortex. Proceedings of the National Academy of Sciences, 99, 4115-4120.

Gray, J. R., Chabris, C. F., \& Braver, T. S. (2003). Neural mechanisms of general fluid intelligence. Nature Neuroscience, 6, 316-322.

Gray, J. R., \& Thompson, P. M. (2004). Neurobiology of intelligence: Science and ethics. Nature Reviews Neuroscience, 5, 471-482.

Green, R. (1984). Preferred stimulation levels in introverts and extraverts: Effects on arousal and performance. Journal of Personality \& Social Psychology, 46, 1303-1312.

Haier, R. J., Siegel, B., Tang, C., Abel, L., \& Buchsbaum, M. S. (1992). Intelligence and changes in regional cerebral glucose metabolic-rate following learning. Intelligence, 16, 415-426.

Haier, R. J., White, N. S., \& AlKire, M. T. (2003). Individual differences in general intelligence correlate with brain function during nonreasoning tasks. Intelligence, 31, 429-441.

HefFernan, T. M., \& Ling, J. (2001). The impact of Eysenck's extraversion-introversion personality dimension on prospective memory. Scandinavian Journal of Psychology, 42, 321-325.

HocKeY, G. R. J. (1997). Compensatory control in the regulation of human performance under stress and high workload: A cognitiveenergetical framework. Biological Psychology, 45, 73-93.

Humphreys, M. S., \& Revelle, W. (1984). Personality, motivation, and performance: A theory of the relationship between individual differences and information processing. Psychological Review, 91, 153-184. Kumari, V., fFytche, D. H., Williams, S. C., \& Gray, J. A. (2004). 
Personality predicts brain responses to cognitive demands. Journal of Neuroscience, 24, 10636-10641.

Larsen, R. J., \& Ketelaar, E. (1991). Personality and susceptibility to positive and negative emotional states. Journal of Personality \& Social Psychology, 61, 132-140.

LIEBERMAN, M. D. (2000). Introversion and working memory: Central executive differences. Personality \& Individual Differences, 28, 479-486.

Lieberman, M. D., \& Rosenthal, R. (2001). Why introverts can't always tell who likes them: Multitasking and nonverbal decoding. Journal of Personality \& Social Psychology, 80, 294-310.

MacDonald, A. W., Cohen, J. D., Stenger, V. A., \& Carter, C. S. (2000). Dissociating the role of the dorsolateral prefrontal cortex and anterior cingulate cortex in cognitive control. Science, 288, 1835-1838.

Meng, X., Rosenthal, R., \& Rubin, D. B. (1992). Comparing correlated correlation coefficients. Psychological Bulletin, 111, 172-175.

Neubauer, A. C., Fink, A., \& Schrausser, D. G. (2002). Intelligence and neural efficiency: The influence of task content and sex on the brain-IQ relationship. Intelligence, $\mathbf{3 0}, 515-536$.

Paus, T. (2001). Primate anterior cingulate cortex: Where motor control, drive and cognition interface. Nature Reviews Neuroscience, $\mathbf{2}$ 417-424.

Paus, T., Koski, L., Caramanos, Z., \& Westbury, C. (1998). Regional differences in the effects of task difficulty and motor output on blood flow response in the human anterior cingulate cortex: A review of 107 pet activation studies. NeuroReport, 9, R37-R47.

Pochon, J. B., Levy, R., Fossati, P., Lehericy, S., Poline, J. B., PilLON, B., Le Bihan, D., \& Dubois, B. (2002). The neural system that bridges reward and cognition in humans: An fMRI study. Proceedings of the National Academy of Sciences, 99, 5669-5674.

Raven, J., Raven, J. C., \& Court, J. H. (1998). Manual for Raven's progressive matrices and vocabulary scales. Oxford: Oxford Psychologists Press.

Rusting, C. L., \& LARSEN, R. J. (1999). Clarifying Gray's theory of personality: A response to Pickering, Corr, and Gray. Personality \& Individual Differences, 26, 367-372.

Rypma, B., \& D'Esposito, M. (1999). The roles of prefrontal brain regions in components of working memory: Effects of memory load and individual differences. Proceedings of the National Academy of Sciences, 96, 6558-6563.

Simpson, J. R., Drevets, W. C., Snyder, A. Z., Gusnard, D. A., \& RaIChle, M. E. (2001). Emotion-induced changes in human medial prefrontal cortex: II. During anticipatory anxiety. Proceedings of the National Academy of Sciences, 98, 688-693.

Stelmack, R. M. (1990). Biological basis of extraversion: Psychophysiological evidence. Journal of Personality, 58, 293-311.

SutTon, S. K., \& Davidson, R. J. (1997). Prefrontal brain asymmetry: A biological substrate of the behavioral approach and inhibition systems. Psychological Science, 8, 204-210.

Talairach, J., \& Tournoux, P. (1988). Co-planar stereotaxic atlas of the human brain. New York: Thieme.

Wilkinson, D., \& HaLligan, P. (2004). The relevance of behavioural measures for functional-imaging studies of cognition. Nature Reviews Neuroscience, 5, 67-73.

Yerkes, R. M., \& Dodson, J. D. (1908). The relation of strength of stimulus to the rapidity of habit formation. Journal of Comparative Neurology \& Psychology, 18, 459-482.

Zald, D. H., Mattson, D. L., \& Pardo, J. V. (2002). Brain activity in ventromedial prefrontal cortex correlates with individual differences in negative affect. Proceedings of the National Academy of Sciences, 99, 2450-2454.

Zelenski, J. M., \& Larsen, R. J. (1999). Susceptibility to affect: A comparison of three personality taxonomies. Journal of Personality, 67, 761-791.

(Manuscript received October 8, 2004; accepted for publication March 23, 2005.) 